\title{
Underwater Multi-influence Measurements \\ as a Mean to Characterize the Overall Vessel \\ Signature and Protect the Marine Environment
}

Medidas multi-influencia como medio para caracterizar la firma global de un buque y proteger el entorno marino

Antonio Sánchez García ${ }^{1}$

Adolfo Hernández Solano ${ }^{2}$

Francisco J. Rodrigo Saura ${ }^{3}$

Patricio Muñoz Esparza ${ }^{4}$

\begin{abstract}
The overall signature of a vessel comprises acoustic, magnetic, electric-field, pressure, and seismic radiations. Over the past years, the international community's efforts have mainly centered on reducing the acoustic influence with the dual aim of decreasing the vessel's detectability and reducing the levels of acoustic pollution generated in the marine environment. Nowadays, the need to act not only on the acoustic radiation but against the overall set of the vessel's radiations is becoming increasingly clear, both in the military and the civilian fields, based on aspects like vessel stealthiness, security of harbor and critical infrastructures, and environmental protection. As a key element to achieve this goal, it is greatly important to have at our disposal highly modular and adaptable measurement systems covering the overall set of the vessel's radiations, with a high capacity of data transmission to base centers to have the capacity to make measurements in all kinds of marine environments.
\end{abstract}

Key words: vessel signature, multi-influence, underwater measurement, environmental protection, portable systems

\section{Resumen}

La firma global de un buque está compuesta por radiaciones: acústica, magnética, de campo eléctrico, de presión y sísmica. A lo largo de los últimos años, los esfuerzos de la comunidad internacional se han centrado principalmente en reducir la influencia acústica, con el doble objetivo de disminuir la detectabilidad del buque y reducir el nivel de contaminación acústica generada en el entorno marino. En la actualidad, se está constatando con claridad la necesidad de actuar no sólo sobre la radiación acústica, sino sobre el conjunto global de las radiaciones del buque, tanto en el ámbito militar como en el ámbito civil, basado en aspectos como: la discreción de los buques, la seguridad en puertos e infraestructuras críticas y la protección ambiental. Como elemento clave para alcanzar esta meta, es de gran importancia tener a nuestra disposición sistemas de medida adaptables y altamente modulares que cubran el conjunto total de las radiaciones de los buques, con alta capacidad de transmisión de datos a centros base, con el objeto de tener la capacidad de realizar medidas en toda clase de entornos marinos.

Palabras claves: firma global del buque, multi-influencia, medición bajo agua, protección ambiental, sistemas portátiles

Date Received: January 17th, 2013 - Fecha de recepción: 17 de Enero de 2013

Date Accepted: February 12th, 2013 - Fecha de aceptación: 12 de Febrero de 2013

\footnotetext{
${ }^{1}$ Joint-stock company of Submarine Electronics (SAES). Line of activity of I+D+i. Cartagena, España. e-mail: a.sanchez@electronica-submarina.com

${ }_{2}^{2}$ Joint-stock company of Submarine Electronics (SAES). Commercial management and of Marketing. Cartagena, España. e-mail: a.hernandez@electronica-submarina.com

${ }^{3}$ Joint-stock company of Submarine Electronics (SAES). Systems Engineering Department. Cartagena, España. e-mail: f.rodrigo@electronica-submarina.com

${ }^{4}$ Joint-stock company of Submarine Electronics (SAES). Systems Engineering Department. Cartagena, Espańa. e-mail: p.munoz@electronica-submarina.com
} 


\section{Introduction}

All vessels, independent of their shape and size, emit onto the sea a set of radiations that make up the socalled vessel signature. This signature characterizes and identifies univocally the vessel the same way the fingerprint identifies human beings. The importance of this signature is well-known since early in the past century, mainly in the defense field and specially centered on the so-called acoustic and magnetic signatures. For example, detection of vessels based on their acoustic signatures was quite important in the naval field during World War II.

In parallel with technological improvements during the 20th century, and especially in the defense field, specific techniques have been developed to reduce the level of radiations emitted onto the sea by vessels. At the beginning, reduction techniques were limited to the acoustic radiation. Next, magnetic radiation was also taken into consideration and, more recently, the electric-field, pressure, and seismic radiations have also been considered of interest. The group of five radiations referred to above make up the so-called multiinfluence signature of a vessel. An example of the simulated electric signature radiated by a sweep gear is shown in Fig. 1.

Monitoring the multi-influence signature is greatly important in the defense field and in the case of vessels like submarines it becomes a matter of survival. Also, it is becoming increasingly important in the civilian field related to the marine environment preservation, especially in the case of the marine fauna living in this environment, due to the influence of these radiations on their behavior. Finally, it is worth noting that detection of this signature permits us to determine the presence of threats in harbors/ports, critical infrastructures or cultural assets located on the sea floor, making it possible to implement specific actions to neutralize these threats.

This paper comprises four sections in addition to this introduction section: the first section describes the main characteristics of the radiations that make up the multi-influence signature. In the next two sections, the importance of this signature in the defense and civilian fields is analyzed. In the following section, a system especially adapted to measure multi-influence signatures is described. Finally, the paper is completed with the conclusions of the study.

\section{Characteristics of the Multi- influence signature of a vessel}

As previously stated, the multi-influence signature of a vessel comprises five types of radiation: acoustic, magnetic, electric, pressure, and seismic. Each incorporates specific characteristics. Then, a brief description of their main characteristics is presented.

Fig. 1. Simulation of the electric field radiated by a sweep gear
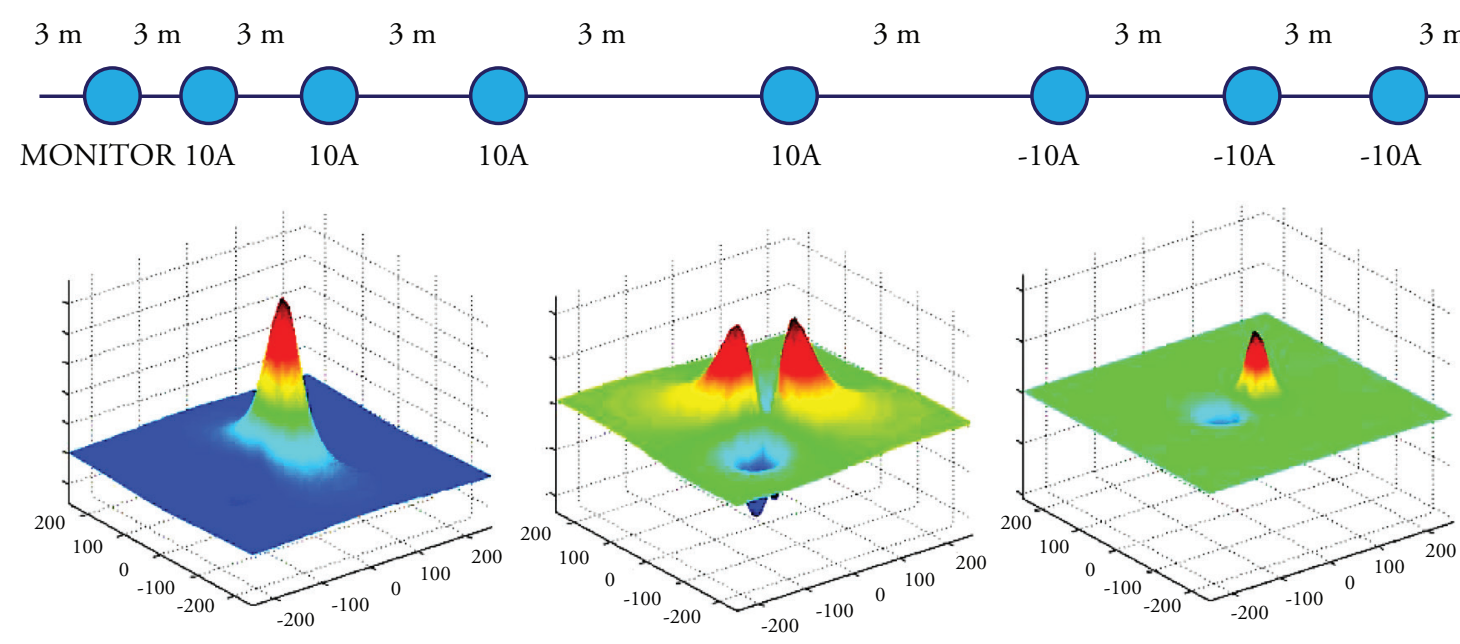
Fig. 2. Underwater sound propagation paths obtained from an acoustic propagation model

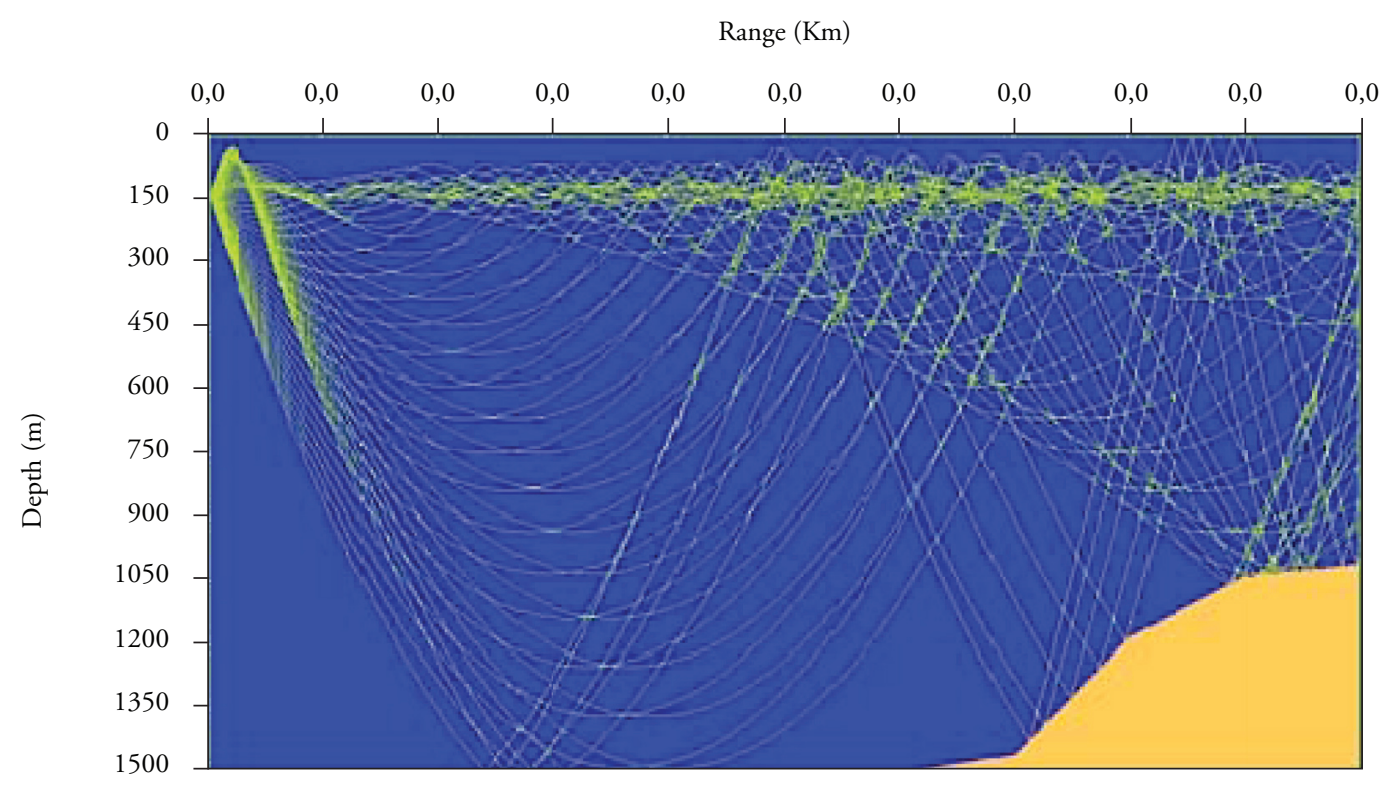

\section{Acoustic radiation}

The sound generated by a vibrating source is propagated as a wave in an elastic medium such as the sea, originating pressure changes that are susceptible of being measured.

The underwater sound propagation, characterized by its high performance, being the way radiation currently best propagates through this medium [1], reaching long distances in the case of low frequencies.

Vessels emit two types of generic signals: broadband and narrowband. The former is characterized by covering a wide spectrum of frequencies, meanwhile the latter is limited to a narrow spectrum.

There are different sound sources in vessels. The three main are: machinery noise, propeller noise, and hydrodynamic noise. A scheme of underwater sound propagation obtained from an underwater acoustic propagation model is shown in Fig. 2.

\section{Magnetic radiation}

The Ship's magnetic influence comprises two components: the static component (SM) and the alternating one (AM). The static component is generated by permanent and induced magnetic fields.

The ship's permanent magnetic field is due to the magnetization of its construction magnetic materials by the Earth's magnetic field. Besides, the Earth's magnetic field, as an external magnetic field, always contributes to the ship's magnetic signature. This component depends on the ship's course and localization of the area.

In addition to permanent and induced magnetic fields, the Corrosion Related Magnetic (CRM) field also contributes to the static magnetic component of the signature. This field is due tto the existence of corrosion currents through the sea water, which has an associated magnetic field.

The alternating component of magnetic signature is generated by:

- Currents in the rotating coils of ship turbines. These coils perform as magnetic dipoles, which generate AC magnetic signature.

- Sea water Foucault currents induced by the magnetic dipoles. These currents are time varying and are also associated with alternating electric fields.

- Electric currents flowing through the ship's 
hull due to electric equipment failures or inadequate design.

- Inherent magnetic field radiated by any rotating electric machinery in the ship.

Besides, power supply ripple generates alternating currents through the water. An example of simulation of the magnetic field generated by a vessel is shown in Fig. 3.

Fig. 3. Simulation of magnetic field generated by a vessel

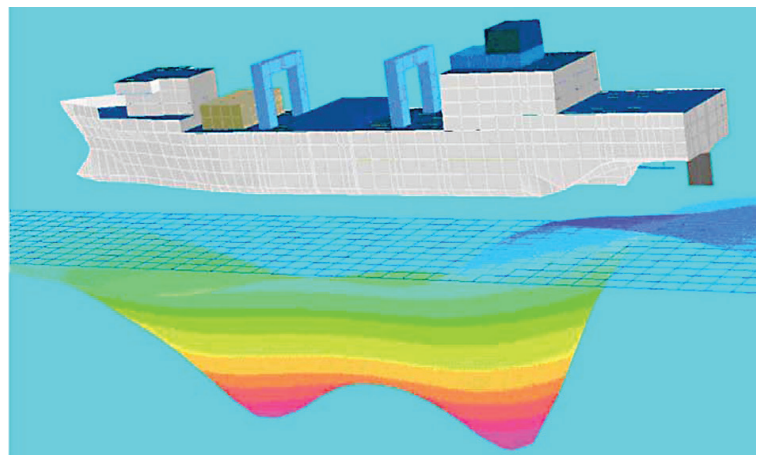

\section{Electric-field radiation}

The ship's electric signature is composed by two components: the static component, which is the Underwater Electric Potential (UEP) and the alternating component, or Extremely Low Frequency Electric (ELFE).
The static UEP component represents the near field influence and its temporal variation depends on the ship's speed and size. The static electric signature of a ship is due to the electric currents generated by the galvanic corrosion process. In order to avoid this corrosion, cathodic protection systems are used. Two types of cathodic protection systems exist: passive and active or Impressed Current Cathodic Protection (ICCP). Passive systems use sacrifice anodes, whereas active systems use impressed current anodes and reference electrodes. Quite often, cathodic protection systems contribute drastically to electric signature and constitute the main generator for this influence.

The alternating ELFE component covers a bandwidth of approximately $3 \mathrm{kHz}$ and represents the near and far field influence. The ELFE component is due to the following factors:

1. Modulation of the corrosion current: galvanic current is modulated due to the spin of blades and propellers.

2. Ripple in machinery power supply of the ship. A frequency tone appears, corresponding to the power supply frequency.

3. Ripple in degaussing ICCP systems, corresponding to the modulation suffered by the ICCP system current due to variations

Fig. 4. Example of simulation of the electric signature radiated by a vessel
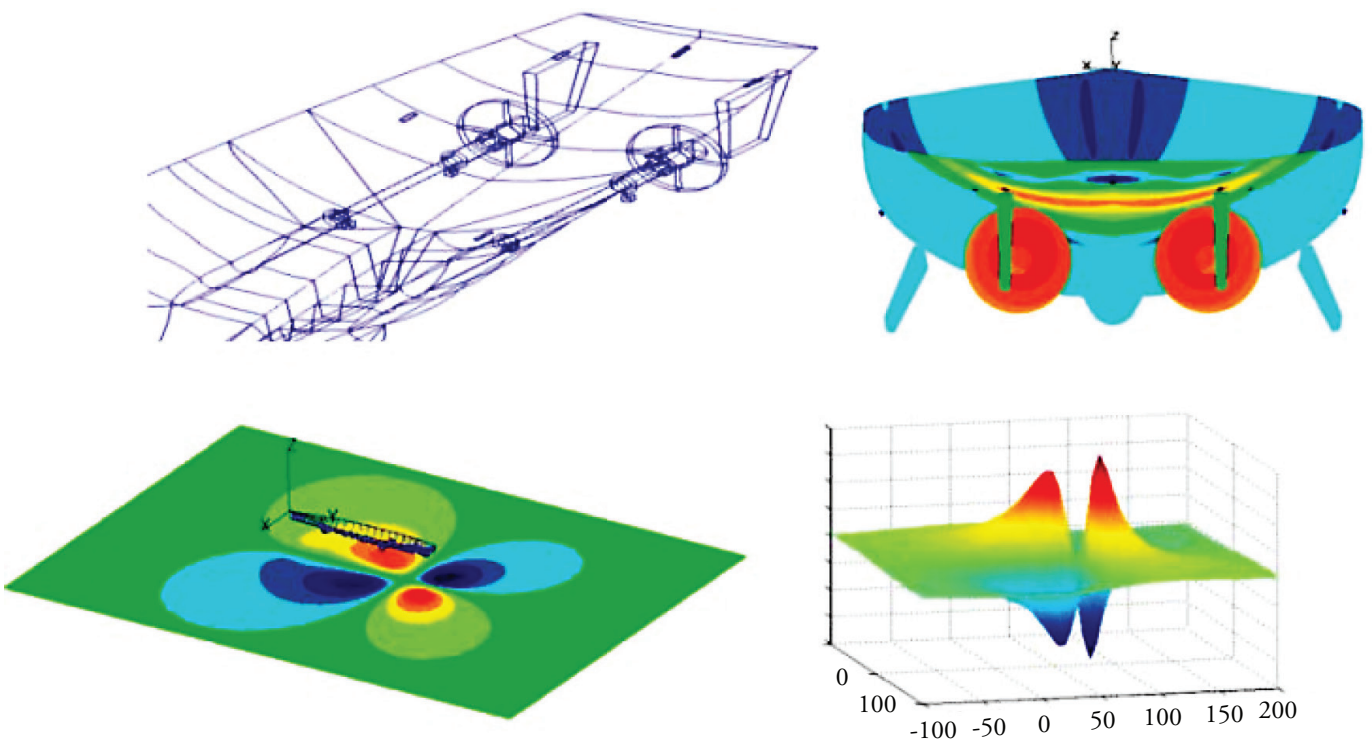
in the resistance between the shaft and hull of the ship. Fig. 4 shows an example of simulation of the electric signature radiated by a vessel.

\section{Pressure radiation}

Hydrostatic pressure due to water depth varies slowly with atmospheric pressure changes and tide rising and falling. Besides, it can vary fast with waves and train of waves, or with a ship. Pressure variation due to a ship movement is usually very small. This small variation constitutes the ship's pressure signature and it is produced by the Bernoulli Effect of the water flowing from bow to stern. This flow originates a pressure increase at the ship's bow and stern and a decrease in the central zone (suction), whose peak is directly proportional to ship speed and its underwater shape. Fig. 5, shows this pattern in a simulation of a ship's pressure signature. Therefore, induced pressure fluctuations by the ship are superimposed with the nominal static pressure from the bottom and natural disturbances caused by tide, waves and swells.

\section{Seismic radiation}

Seismic influence is generated by the same sources as for the acoustic influence. When an acoustic wave reaches a surface, the majority of the energy is reflected but a percentage is absorbed by the new medium. Thus, very low frequency (below $10 \mathrm{~Hz}$ ) acoustic signals propagate up to the sea floor and transmit through it as a seismic perturbance. This type of perturbance travels much faster through the sea floor than through sea water.

From this seismic point of view, it is obvious that the acoustic energy penetrating into the sea floor may sometimes contribute considerably to medium-range and long-range acoustic trans mission. One clear example that shows seismic influences behavior is the existence of a critical frequency. For all frequencies below this limit, absorption phenomena in incident waves appears, and this phenomena depends on the characteristics of the materials and layers of the sea floor.

Fig. 6. Ship seismic signature physical phenomena

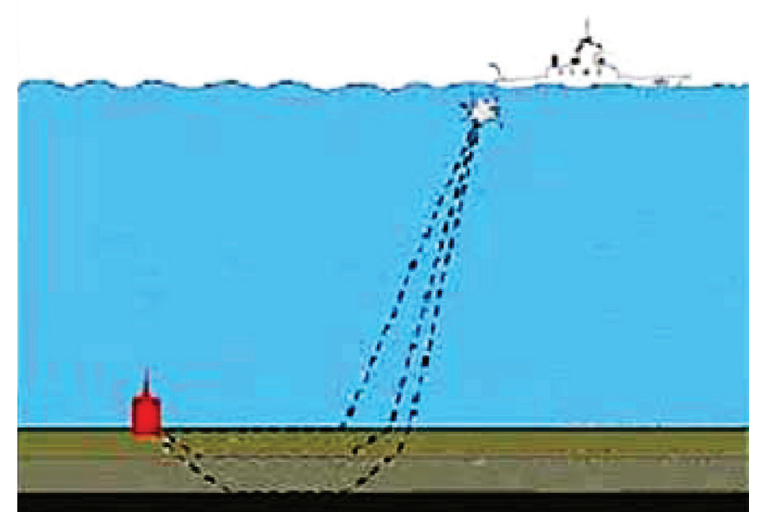

Fig. 5. EShip's Pressure Signature Simulation

PRESSURE $($ Speed $=9,3 \mathrm{~m} / \mathrm{s} ;$ Depth $=35 \mathrm{~m})$

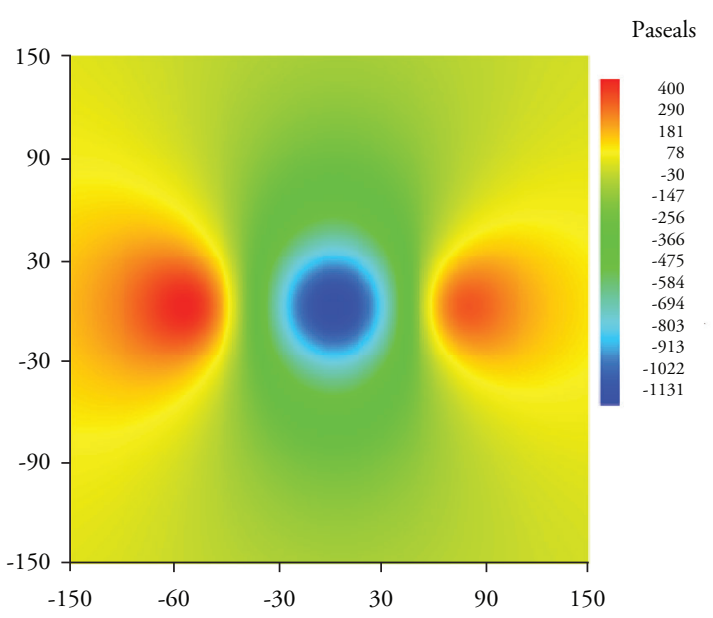

PRESSURE $($ Speed $=14,4 \mathrm{~m} / \mathrm{s} ;$ Depth $=35 \mathrm{~m})$

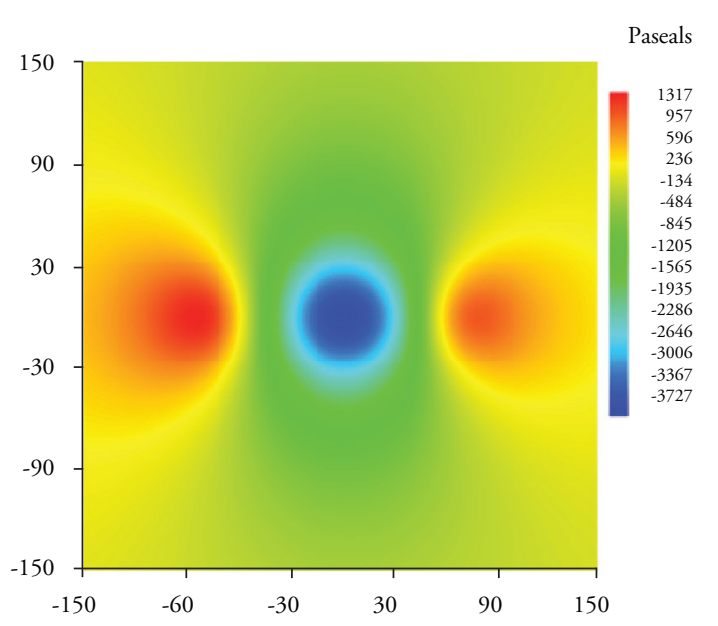




\section{The multi-influence signature in the defense field}

Intelligence databases have become elements of great importance for the navies in different countries. These databases contain, as distinctive data, the signatures of the vessels. At the beginning, they contained acoustic data and sometimes magnetic data. Currently, they also seek to incorporate data corresponding to the rest of influences. These databases permit discriminating not only the type and class of vessel, but also the specific unit of the class, providing a considerable tactical advantage.

Currently, the trend in recent decades relative to the reduction of the vessel's signature is being accentuated. This trend is particularly intense in the case of submarines. They seek to increase their level of protection by increasingly becoming more stealth vessels as a means to counter-act the development of increasingly intelligent weapons like last-generation torpedoes. Fig. 7 shows an image of U-206 type submarines.

Fig. 7. U-206 class submarines : A.R.C. Intrépido and A.R.C. Indomable

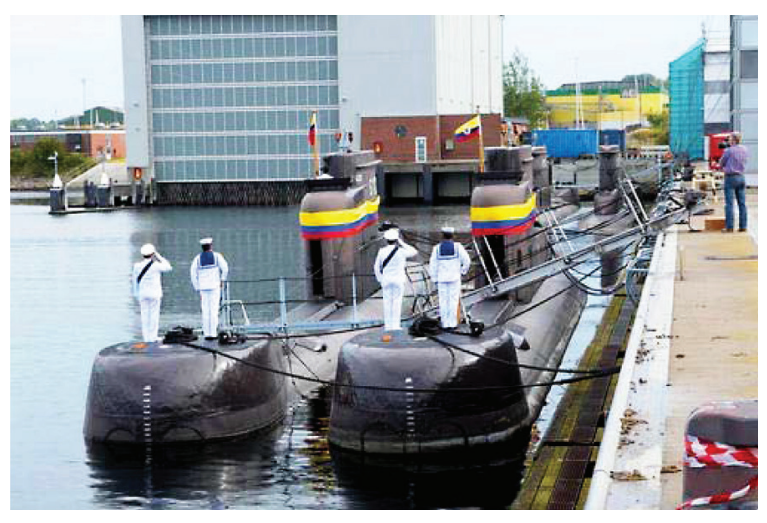

In parallel with that stated above, more sophisticated systems incorporating a wide range of sensors are now being developed. The use of these multi-influence systems permits vessels to significantly increase their detection capacities from the combination of the data provided by their suite of sensors, enabling a considerable reduction in the number of false alarms. Also, in the defense facilities protection field, the use of these multi-influence sensors has permitted significantly increasing their security. This fact is based on early and more accurate detection of threats coming from the marine environment, such as: divers moving autonomously, manned and unmanned underwater vehicles (SDV, ROV, UUV), mini-submarines, etc.

\section{The multi-influence signature in the civilian field}

During recent years, growing awareness has emerged worldwide on the need to protect the marine environment, especially from human activities like: fishing, sailing, harbor works, or seismic oil and gas explorations that convey significant increase in the level of a range of pollutants such as acoustic noise and other sources of energy, including electric and magnetic sources. This growing awareness has entailed the development of a range of national and international regulations focused on achieving effective preservation of the marine environment. Among these regulations is the Marine Strategy Framework Directive, promulgated by the European Union in 2008, which introduced a set of qualitative descriptors to determine the good environmental status. One of these descriptors states that: "the introduction of energy, including underwater noise, is at levels that do not adversely affect the marine environment". The effective application of this Directive, and of other regulations related with the marine environment protection, implies both measurement of the level of energy radiations emitted to the sea [2] and detection of marine fauna presence in specific areas on which high energy levels are detected with the aim of protecting this fauna from the potential harmful effects of the radiated energy.

This detection is mainly based on acoustic sensors, although other alternative detection means are currently being analyzed, such as detection of marine fauna based on the alteration of the underwater electric or magnetic fields originated by their presence. In Fig. 8, two kinds of cetacean species: bottlenose dolphin and sperm whale are shown; both are endangered species. 
Fig. 8. Species of cetaceans: bottlenose dolphin (left) and sperm whale (right)
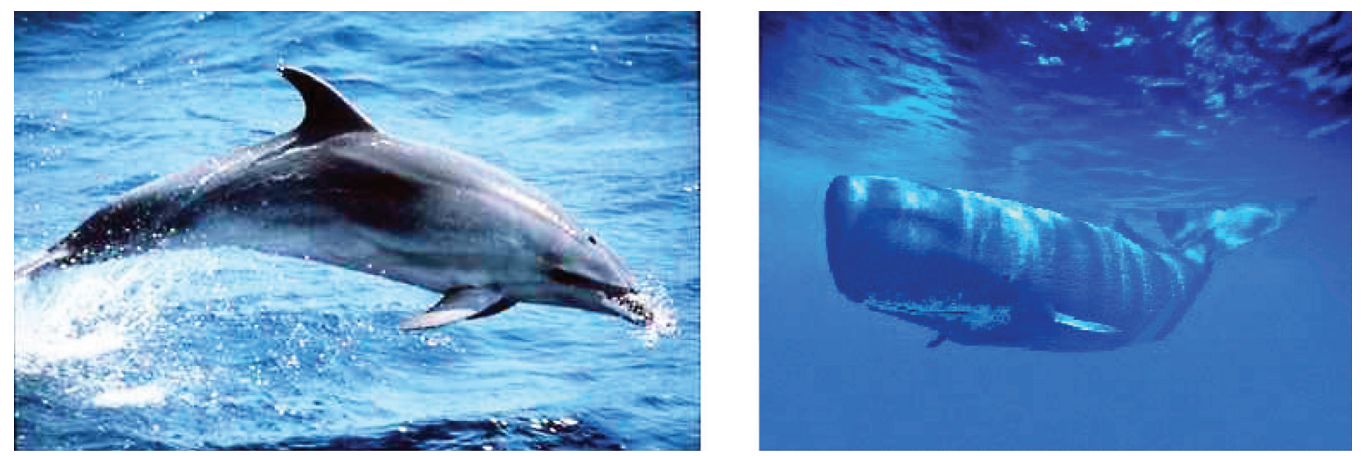

Just like in the defense field, use of multiinfluence sensor systems provides an effective protection of harbors and critical infrastructures like oil refineries and thermal power stations against hostile intruders. This protection can also be extended to other fields of remarkable interests as is the case of marine reserves, ship wrecks, or underwater archaeological remains.

\section{The multi-influence signature measurements}

With regards to multi-influence measurements, the trend in the civilian field is to advance towards a standardization process in the procedures and parameters of the measurements. These standards have long existed in the defense field. The precursor of this process is, again, the acoustic influence for which a standard has recently (2009) been developed by the Acoustical Society or America (ASA): "Quantities and Procedures for Description and Measurement of Underwater Sound from Ships". Additionally, within the European Union scope specific programs exist focused on the definition of a European standard. Measurements are usually normalized to common references in order to compare these coming from different systems, as is the case of the 1-meter from the source reference for acoustic measurements or a common reference point for all the vessels in the case of measurements of electric and magnetic fields.

The fact that multi-influence sensors provide different detection ranges permits establishing different detection layers as a function of the sensor range. Detection ranges depend on both the specific characteristics of the marine environment and those of the vessel being tested and the sensors used. In a first approach, it can be stated that both acoustic and seismic sensors provide detection distances in the range of kilometers, being the seismic sensor especially dependent on the characteristics previously referred to, electric-field and magnetic sensors in the range of hundreds of meters and pressure sensors in the range of tens of meters.

The wide range of operational environments in which multi-influence measurements are susceptible to be taken makes it highly advisable to have at our disposal modular and portable systems with small dimensions and weights. These kinds of systems permit their deployment and recovery in different marine areas within a reduced time interval and without requiring complex means. Other aspects to be stressed are: the capacity of data transmission to base centers (located on shore or onboard vessels) with an adequate bandwidth to cover the characteristics of the signatures tested and the capacity of storing and processing the measured influences, focused on providing accurate and useful information to the system operator. An example of a system that complies with the characteristics previously described is the multiinfluence measuring system (MIRS) developed by SAES (see underwater units deployed on the sea bottom in Fig. 9). 
Fig. 9. Underwater units of the multi-influence measurement system (MIRS) developed by SAES

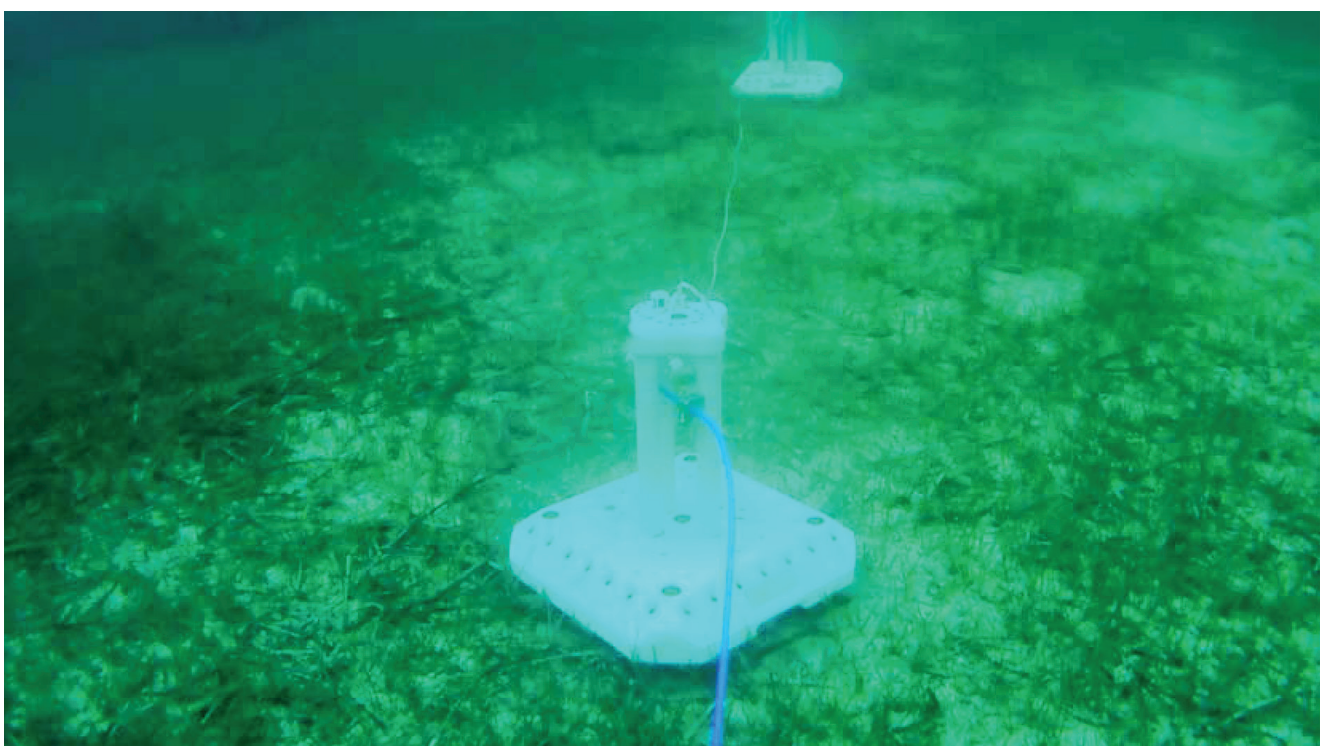

The MIRS has been tested in operational environments, showing its versatility, ease of use, and accuracy taken as reference calibrated systems. In Fig. 10, graphic outputs of the multiinfluence signature of a merchant vessel are shown.

\section{Conclusions}

All vessels, when sailing through the sea, radiate a set of influences (acoustic, magnetic, electric, pressure, and seismic) that make up their multi- influence signature. Some of these influences (acoustic and magnetic) have been measured since decades ago to characterize vessels in the defense field and to monitor the acoustic pollution in the civilian field. Recently, interest in the international community on having at its disposal the overall signature of the vessels has emerged, seeking to globally evaluate its impact on the marine environment.

In the defense field, from the fleet's point of view to have at our disposal multi-influence data from vessels permits performing specific

Fig. 10. Set of measurements of influences of a merchant vessel taken by the MIRS developed by SAES

Acoustic

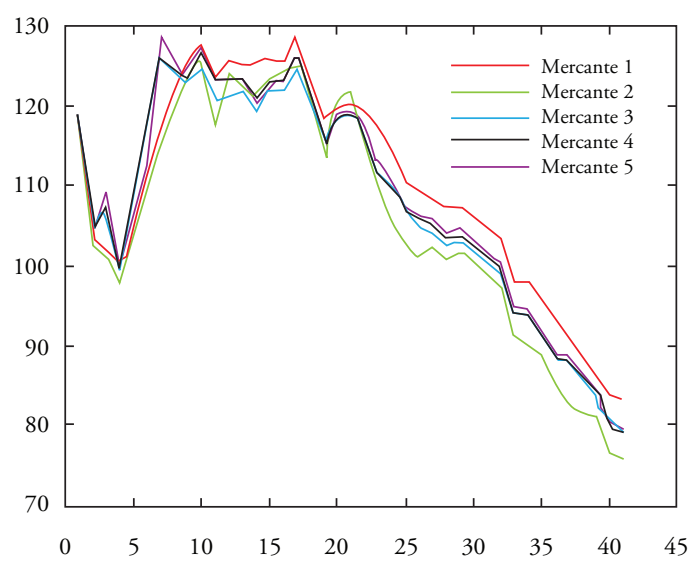

Seismic

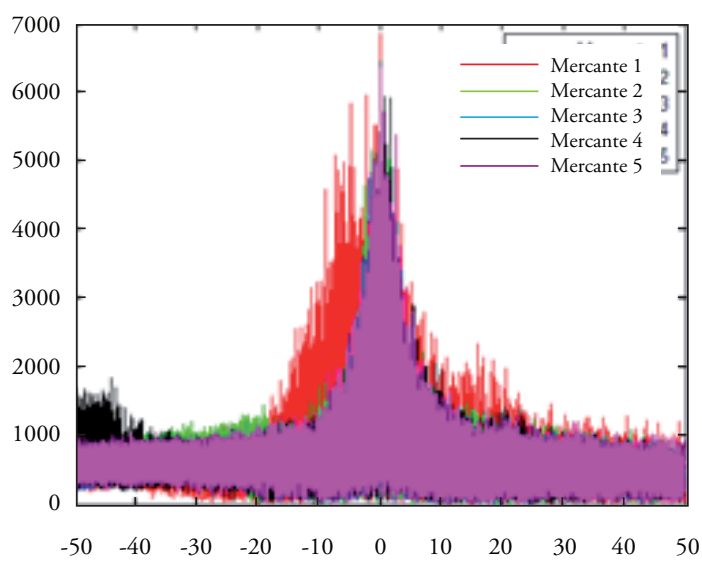


Magnetic

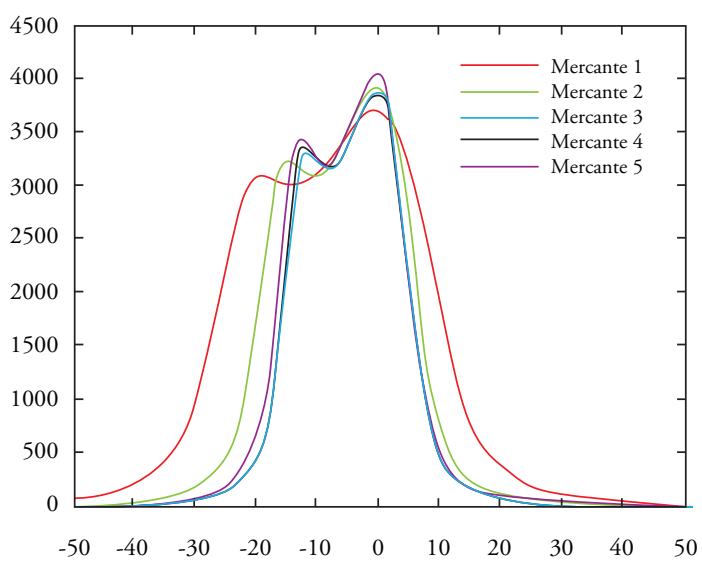

Electric

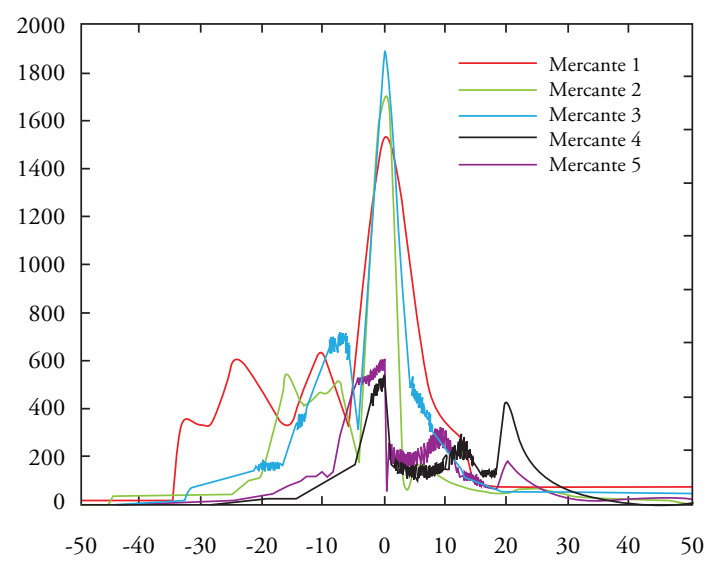

Pressure

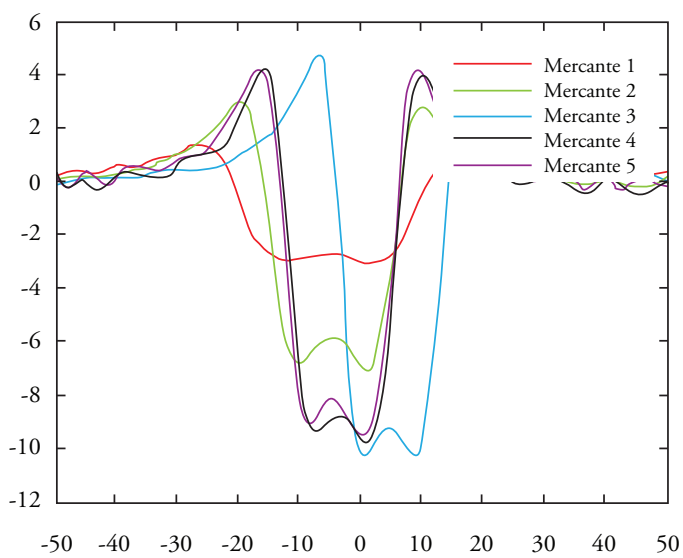

tasks and studies focused on reducing its own signature to decrease the probability of being detected. On the other hand, from the point of view of threats, this data permits characterizing the vessel's signature with the aim of increasing our capacity to detect them.

In the civilian field, interest is centered on the marine environment preservation, especially of its marine fauna. In the dual defense-civilian field, protection systems based on multiinfluence sensors constitute a highly efficient means to detect hostile intruders.

Due to the variety of operational areas in the marine environment, it is highly advisable to have at our disposal modular systems with contrasted capacities of data transmission, recording the measurements and processing focused on providing relevant information to the system operator. The MIRS developed by SAES configures as a verified and in-service system than complies with the requirements established for the multi-influence measurement of all kinds of platforms or naval devices in the whole spectrum of operational environments.

\section{References}

[1] R. J. URICK. Principles of Underwater Sound. McGraw-Hill (1993).

[2] W. J. RICHARDSON, C. R. GREEN, C. I. MALME, D. H. THOMSON. Marine mammal and noise. Academic Press Inc. (1995). 